\title{
Risk of non-alcoholic fatty liver disease in patients with chronic plaque psoriasis: an updated systematic review and meta-analysis of observational studies
}

\author{
F. Bellinato ${ }^{1}$ (1) $\cdot$ P. Gisondi ${ }^{1}$ - A. Mantovani ${ }^{2} \cdot$ G. Girolomoni ${ }^{1} \cdot$ G. Targher $^{2}$
}

Received: 25 November 2021 / Accepted: 25 January 2022 / Published online: 11 February 2022

(c) The Author(s) 2022

\begin{abstract}
Purpose Chronic plaque psoriasis is associated with the presence of non-alcoholic fatty liver disease (NAFLD), but the magnitude of this association remains currently uncertain. We aimed to investigate the magnitude of the association between psoriasis and the risk of prevalent and incident NAFLD, and to assess whether psoriasis severity and/or psoriatic arthritis are associated with a greater risk of NAFLD.

Methods A systematic review and meta-analysis of observational studies evaluating the association between psoriasis and NAFLD, as diagnosed by imaging or International Classification of Diseases codes was performed. Literature search on PubMed, Scopus and Web of Science on May 3, 2021 was undertaken. Studies using liver biopsy were not available. For the meta-analysis, the random-effects modelling was adopted.

Results We identified 15 observational (case-control and cross-sectional) studies for a total of 249,933 patients with psoriasis (49\% with NAFLD) and 1,491,402 controls (36\% with NAFLD). Psoriasis was associated with prevalent NAFLD $\left(n=11\right.$ studies; pooled random-effects odds ratio [OR] 1.96, 95\% CI 1.70-2.26; $\left.I^{2}=97 \%, p<0.01\right)$. Psoriatic patients with NAFLD had a higher mean psoriasis area and severity index (PASI) than their counterparts without NAFLD $(n=8$ studies, pooled weighted mean difference: $3.93,95 \% \mathrm{CI} 2.01-5.84 ; I^{2}=88 \%, p<0.01$ ). The risk of NAFLD was marginally higher in patients with psoriatic arthritis than in those with psoriasis alone ( $n=5$ studies, pooled random-effects OR $1.83,95 \%$ CI $0.98-3.43 ; I^{2}=64 \%, p=0.03$ ). Sensitivity analyses did not alter these findings. Funnel plot did not show any significant publication bias. A major limitation of the study was the high degree of heterogeneity across studies.

Conclusion Psoriasis is associated with prevalent NAFLD and this risk parallels the severity of psoriasis.
\end{abstract}

Keywords Psoriasis $\cdot$ NAFLD $\cdot$ Meta-analysis $\cdot$ MAFLD $\cdot$ Comorbidities $\cdot$ Psoriatic arthritis $\cdot$ Metabolic syndrome

\section{Introduction}

Chronic plaque psoriasis is a common, immune-mediated, inflammatory skin disease affecting nearly $3 \%$ of adults in the general population in Western countries [1]. In the past decade, several observational studies have documented that chronic plaque psoriasis is strongly associated with multiple cardiometabolic co-morbidities, including cardiovascular

F. Bellinato

francesco.bellinato@univr.it

1 Section of Dermatology and Venereology, Department of Medicine, University of Verona, Verona, Italy

2 Section of Endocrinology, Diabetes and Metabolism, Department of Medicine, University of Verona, Verona, Italy disease (CVD), obesity, type 2 diabetes (T2DM) or metabolic syndrome $[1,2]$.

Non-alcoholic fatty liver disease (NAFLD) is considered a purely metabolic liver disease, which includes a spectrum of progressive pathologic liver conditions, ranging from simple steatosis to steatohepatitis (NASH) and cirrhosis [2]. To date, NAFLD has reached epidemic proportions and is the most common cause of chronic liver disease in Western countries (affecting up to $30 \%$ of adults in the general population and up to $\sim 70 \%$ of people with T2DM) [3]. In the last decade, it has become increasingly clear that NAFLD is a 'multisystem' disease [4], which is not only associated with important liver-related complications (such as liver failure or hepatocellular carcinoma [HCC]), but also with extra-hepatic diseases, such as CVD 
[5], chronic kidney disease (CKD) [6] and some types of extra-hepatic cancers [7].

Recently, some observational studies, although not all, have reported that individuals with chronic plaque psoriasis have an increased risk of having NAFLD compared with those without psoriasis [8]. Presently, however, the magnitude of this risk and whether the risk changes with the underlying severity of psoriasis remains uncertain. To our knowledge, there are only two small meta-analyses (published in 2015 and 2019, respectively) that have investigated the association between psoriasis and risk of prevalent NAFLD $[9,10]$. These two small meta-analyses reported that psoriasis is associated with an increased risk of prevalent NAFLD, but the available data on the association of NAFLD with the severity of psoriasis or psoriatic arthritis remain inconclusive. Notably, as discussed in detail below, in the last 2 years, new observational studies examining the association between psoriasis (and its severity) and the risk of NAFLD have been published.

We undertook an updated systematic review and metaanalysis of observational studies to quantify the magnitude of the association between psoriasis and risk of prevalent and incident NAFLD, as well as to examine whether the severity of chronic plaque psoriasis or presence of psoriatic arthritis were associated with a higher risk of prevalent NAFLD.

\section{Methods}

\section{Data sources and searches}

We performed the systematic review according to the Preferred Reporting Items for Systematic Reviews and Meta-Analyses (PRISMA) statement [11]. Since the included studies were observational in design, we followed the reporting items proposed by Meta-analysis Of Observational Studies in Epidemiology (MOOSE) for the meta-analysis of these studies [12]. We conducted a systematic literature search from the inception date to May 3, 2021 through PubMed, Scopus and Web of Science to identify observational studies examining the risk of prevalent NAFLD amongst individuals with and without chronic plaque psoriasis. Search text terms were as follows: (("Arthritis, Psoriatic" [Mesh]) OR ("Psoriasis" [Mesh])) AND ("Non-alcoholic Fatty Liver Disease" OR "NAFLD" [Mesh]). Searches were restricted to human studies. There were no restrictions in terms of sex, race, language, or geographic area. Additionally, we reviewed references from relevant original papers and review articles to identify further eligible studies not covered by the original database searches.

\section{Study selection}

Studies were included in the meta-analysis if they meet the following criteria: (1) observational studies examining the association between chronic plaque psoriasis and risk of NAFLD; (2) studies reporting odds ratios (ORs) with $95 \%$ confidence intervals (95\% CIs) values for the outcome of interest; (3) studies in which the diagnosis of both psoriasis and psoriatic arthritis were based on clinical examinations and specific international criteria, namely CASPAR criteria [13]; (4) studies in which the psoriasis severity was estimated using the psoriasis area and severity index (PASI); and (5) studies in which the diagnosis of NAFLD was based on liver biopsy, imaging techniques or International Classification of Diseases, 9th Revision (ICD-9) or ICD-10 codes, in the absence of significant alcohol consumption or other competing causes for hepatic steatosis [14].

Criteria for exclusion of the studies from the meta-analysis were as follows: (1) congress abstracts, case reports, theses, reviews, commentaries, editorials, or practice guidelines; (2) studies not reporting ORs and 95\% CIs for the outcome of interest; and (3) studies conducted in paediatric population $(<18$ years $)$.

\section{Data extraction and quality assessment}

Data from studies eligible for the aggregate data metaanalysis were extracted by two authors independently (FB and PG). Any disagreements were resolved by consensus and a third author if needed (GG).

For all eligible studies, we extracted information on publication year, study design, study country, sample size, population characteristics, methods used for the diagnosis of psoriasis and NAFLD, psoriasis severity assessed by PASI score, psoriatic arthritis (PsA), matching and confounding factors included in multivariable regression analyses. In case of multiple publications, we included the most up-to-date or comprehensive information. We did not contact any corresponding author of the eligible studies in order to obtain additional information for the meta-analysis.

Two authors (FB and PG) independently assessed the risk of bias. Any discrepancies were addressed by a reevaluation of the original article by a third author (GG). Since the eligible studies were non-randomized, the Newcastle-Ottawa Scale (NOS) was used to judge the quality of the studies included in the meta-analysis, as recommended by the Cochrane Collaboration. Briefly, the NOS uses a star system to evaluate a study in three domains: selection of participants, comparability of study groups, 
and ascertainment of outcomes of interest. We used an adapted version of the NOS for cross-sectional studies. We judged studies that received a NOS score of at least 8 stars to be at low risk of bias, thereby reflecting the highest quality [15].

\section{Data synthesis and analysis}

The primary outcome measure of the meta-analysis was the risk of having NAFLD in patients with chronic plaque psoriasis and non-psoriatic control subjects. The ORs with 95\% CIs were considered as the effect size for all eligible studies. When studies had several adjustment models, we extracted those that reflected the maximum extent of adjustment for potentially confounding risk factors. The adjusted ORs of all eligible studies were then pooled, and an overall estimate of effect size was calculated using the DerSimonian-Laird random-effects model. The psoriasis severity, as assessed by PASI score, in patients with and without NAFLD was displayed as weighted mean difference (WMD) and 95\% CIs for the changes of mean PASI score between psoriatic patients with and without coexisting NAFLD. Visual inspection of the forest plot was used to investigate the possibility of statistical heterogeneity. Statistical heterogeneity was also assessed by the $I^{2}$-statistics, which provides an estimate of the percentage of variability across studies that is due to heterogeneity rather than chance alone. According to Higgins and Thompson [16], $I^{2}$-values of approximately $25 \%$ represent low heterogeneity; approximately $50 \%$ represent medium heterogeneity; and approximately $75 \%$ represent high heterogeneity. Publication bias was evaluated using the funnel plot, the Begg's rank correlation test [17] and the Egger's regression test [18].

To explore the possible sources of heterogeneity among the eligible studies and to test the robustness of the observed associations, we performed subgroup analyses stratifying the eligible studies by study country, methodology used for the diagnosis of NAFLD, NOS scale (i.e. the 'high-quality' studies), or whether they had adjustment at least for age, sex and body mass index (BMI]. Additionally, we performed univariable meta-regression analyses to evaluate the impact of specific moderators (i.e., age, sex and BMI) on the effect size of the risk of having psoriasis-related NAFLD across the eligible studies. We also tested for possible excessive influence of individual studies using a meta-analysis influence test that eliminated each of the included studies one at a time. All statistical tests were two sided and used a significance level of $p<0.05$. We used Review Manager version 5.3 (Copenhagen: The Nordic Cochrane Centre, The Cochrane Collaboration, 2014) and STATA $®$ software v16.1 (StataCorp, College Station, Texas, USA) for all statistical analyses. This systematic review and meta-analysis is registered in PROSPERO, number: CRD42021247549.

\section{Results}

\section{Characteristics of the included articles}

From a total of 76 retrieved articles (after excluding duplicates), a total of 20 eligible studies from PubMed, Scopus or Web of Science databases were identified based on the titles and abstracts, (kappa agreement $=90 \%$ ) (Fig. 1). After full text examination of these 20 potentially eligible studies, we excluded five studies because unsatisfactory inclusion criteria or unacceptable outcome measures, (kappa agreement $=90 \%)($ Supplementary Table 1$)$. After of this exclusion, a total of 15 observational studies were analysed in the meta-analysis. Of these 15 eligible studies, 11 studies were included in the pooled primary analysis that compared the risk of prevalent NAFLD between psoriatic patients and non-psoriatic controls; 8 studies were included in the analysis examining the association between NAFLD and psoriasis severity, as assessed by PASI score; 5 studies were included in the secondary analysis assessing the risk of prevalent NAFLD among psoriatic patients with and without PsA [19-33]. Of note, the study by Ogdie et al. included both a cross-sectional and a cohort design [24].

In particular, the main characteristics of the 11 eligible studies assessing the risk of prevalent NAFLD are reported in Table 1. Overall, these cross-sectional or case-control studies had aggregate data on 249,933 psoriatic patients (mean age 55 years, mean BMI $27.7 \mathrm{~kg} / \mathrm{m}^{2}, 54 \%$ were men, $49 \%$ had NAFLD) and 1,491,402 non-psoriatic healthy controls (mean age 53 years, mean BMI $26.7 \mathrm{~kg} /$ $\mathrm{m}^{2}, 49 \%$ were men, $36 \%$ had NAFLD). Four studies were carried out in the Europe (Italy and the Netherlands), 3 studies were carried out in Asia (Iran and Taiwan) and 4 studies were carried out in the United States. NAFLD was diagnosed by ultrasonography in 7 studies and by ICD$9 / 10$ codes in 4 studies. No studies with liver biopsy data were available for the meta-analysis.

The main characteristics of the 8 eligible studies examining the association between NAFLD and psoriasis severity, as assessed by PASI score, are reported in Table 2. Of these 8 studies, 4 recruited European individuals, 2 studies involved Asian subjects and 2 studies involved United States individuals. NAFLD was diagnosed by imaging techniques (mostly ultrasonography) in all eligible studies, although 2 ones also used biopsy in a small subset of psoriatic patients (for a total of 57 cases). 
Fig. 1 PRISMA flow diagram describing the results of the literature research and study selection

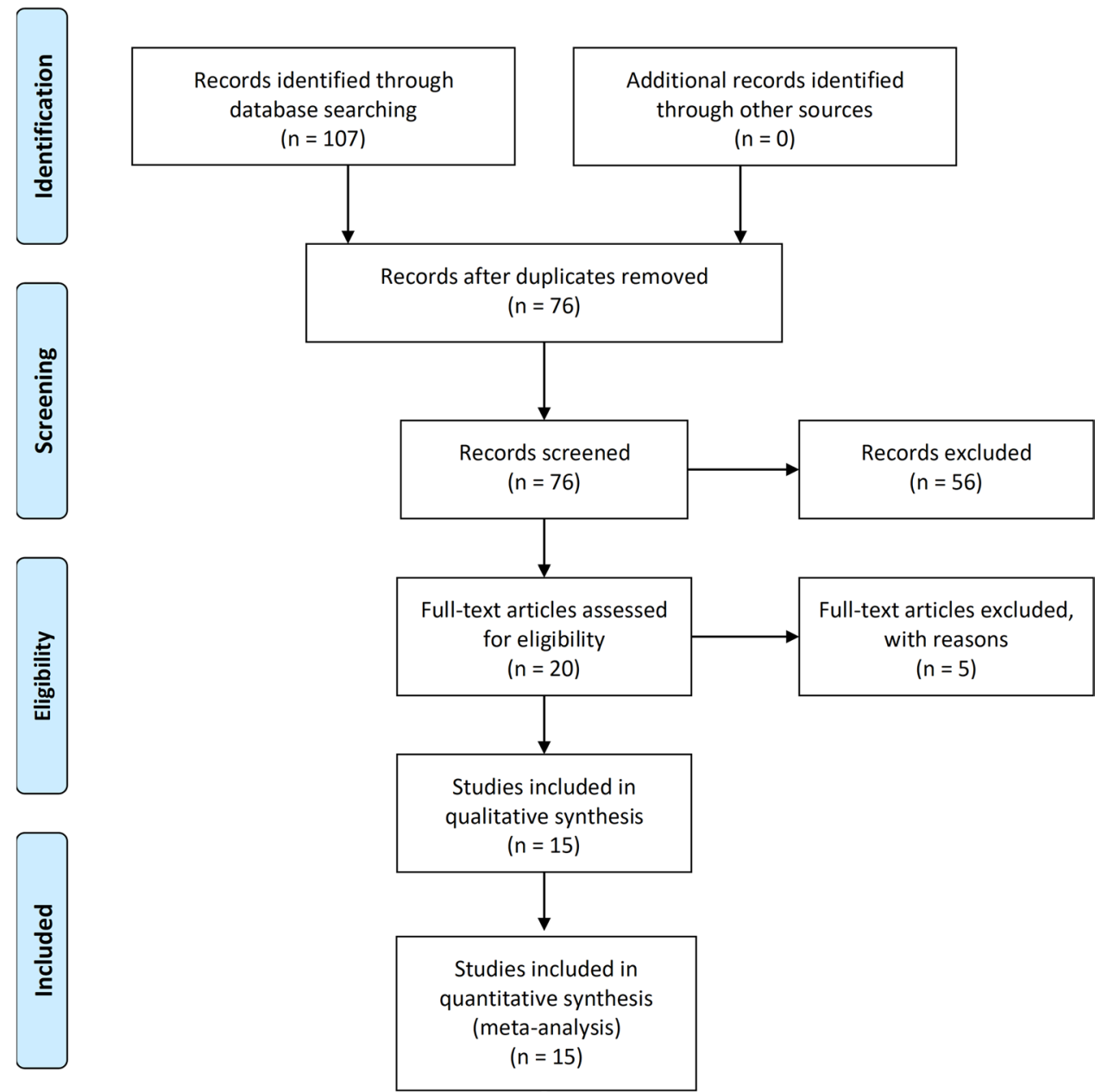

\section{Risk of prevalent NAFLD in patients with and without chronic plaque psoriasis}

The distribution of the 11 eligible studies by estimate of the association between presence of psoriasis and risk of NAFLD, stratified by methods used for NAFLD diagnosis (ultrasonography vs. ICD codes), is plotted in Fig. 2. Overall, patients with chronic plaque psoriasis had an approximately doubled odds ratio for NAFLD compared to non-psoriatic control subjects (pooled random-effects OR $1.96,95 \%$ CI $\left.1.70-2.26, I^{2}=97 \%, p<0.001\right)$. Similar results were observed when the studies were stratified by the methods of NAFLD diagnosis, i.e., either by ultrasonography $(n=7$; pooled random-effects OR 2.02, $95 \%$ CI $1.78-2.28, I^{2}=13 \%, p=0.33$ ) or by ICD codes $(n=4$; pooled random-effects OR $1.83,95 \%$ CI $1.45-2.32$, $\left.I^{2}=97 \%, p<0.001\right)$. Notably, as shown in the figure, there was no significant heterogeneity across the eligible studies that used ultrasonography to diagnose NAFLD $\left(I^{2}=13 \%\right)$.

\section{Psoriasis severity in patients with and without NAFLD}

Figure 3 shows the pooled estimates of mean PASI score among psoriatic patients with and without coexisting NAFLD across 8 eligible studies. Psoriatic patients with NAFLD had significantly greater mean PASI score than their counterparts without NAFLD (pooled WMD: $3.93,95 \%$ CI $\left.2.01-5.84 ; I^{2}=88, p<0.0001\right)$.

\section{Risk of incident NAFLD in patients with psoriasis}

In a subgroup of patients, the study conducted by Ogdie et al. also assessed the risk of incident NAFLD in patients with psoriasis or in those with other autoimmune diseases. In particular, in this study that involved 197,130 patients with psoriasis, 12,308 patients with PsA, 54,251 patients with rheumatoid arthritis and 1,279,754 healthy controls, Ogdie et al. showed that compared to healthy controls, patients 


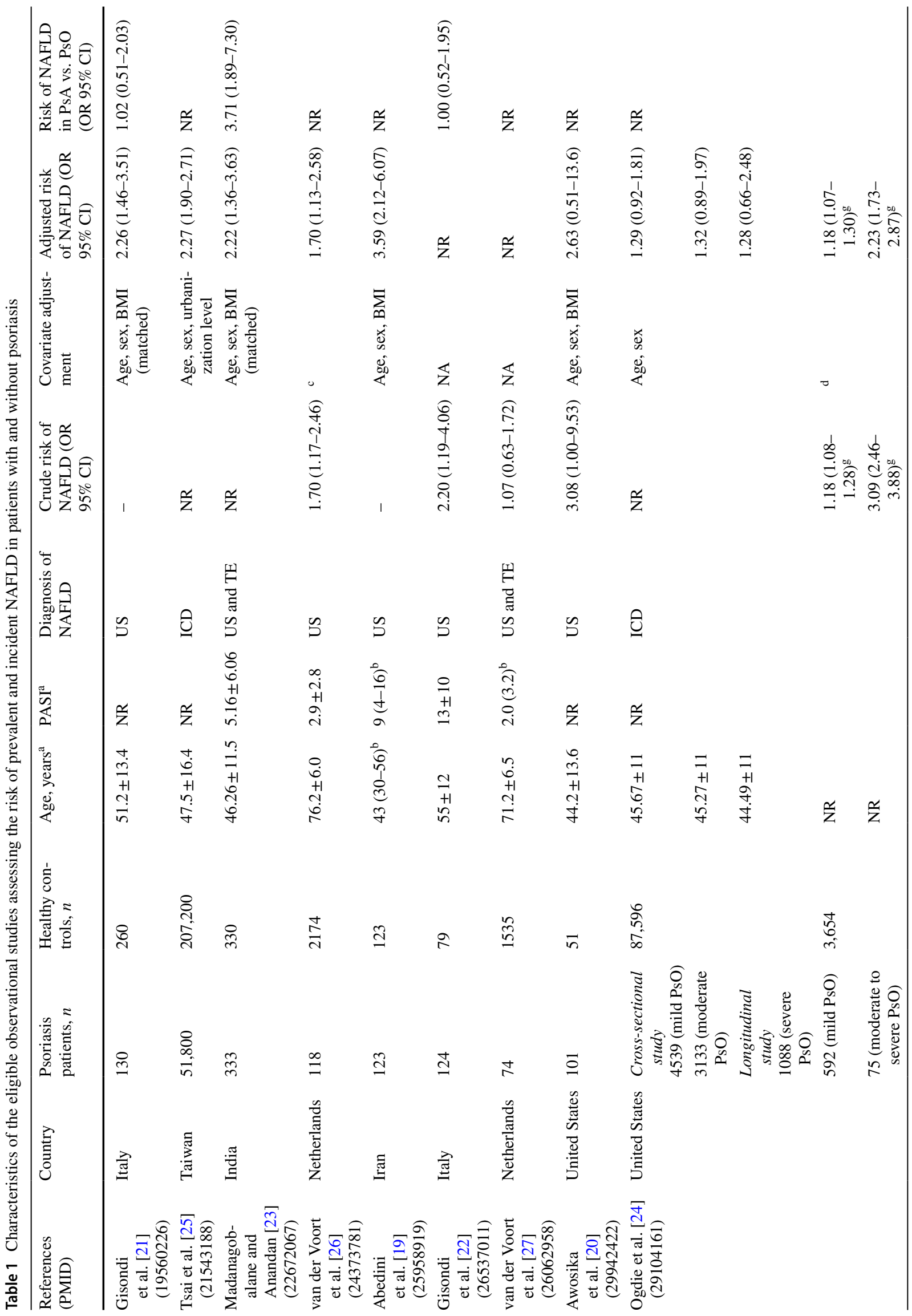


with psoriasis had a higher risk of developing NAFLD (as detected by ultrasonography) over a mean follow-up period of nearly 10 years, even after adjustment for age, sex, BMI and other metabolic risk factors. Notably, this risk increased across severity of psoriasis with an adjusted-hazard ratio of 1.18 [95\% CI 1.07-1.30] for patients with mild psoriasis and adjusted-hazard ratio of 2.23 [95\% CI 1.73-2.87] for patients with moderate to severe psoriasis, respectively [24].

\section{Risk of prevalent NAFLD in patients with and without psoriatic arthritis}

Figure 4 shows the risk of prevalent NAFLD in psoriatic patients with and without coexisting PsA across 5 eligible studies (including a total of 725 psoriatic patients, 514 of whom had PsA). Patients with PsA tended to have a higher risk of NAFLD compared to those without PsA (pooled random-effects OR 1.83 , 95\% CI $0.98-3.43 ; I^{2}=64 \%$, $p=0.061$ ), but this difference did not reach statistical significance.

\section{Subgroup analyses and meta-regressions}

Subgroup analyses were performed to investigate potential causes of heterogeneity across the 11 eligible articles. The stratification of the articles by study country (Supplementary Fig. 1), NOS quality scale (Supplementary Fig. 2), or degree of covariate adjustment (Supplementary Fig. 3) did not influence the association between psoriasis and risk of NAFLD.

We also examined the potential excessive impact of individual studies through an analysis that excluded each of the included studies one at a time (Supplementary Fig. 4). The exclusion of each of the studies from the pooled primary analysis did not affect the overall risk of NAFLD.

Finally, Supplementary Fig. 5 shows the results of univariable meta-regressions. These analyses did not show any significant effects of age $(\beta=-0.01,95 \% \mathrm{CI}-0.03$ to $\left.0.1, R^{2}=33.65 \%\right)$, male sex $(\beta=0.01,95 \% \mathrm{CI}-0.02$ to $\left.0.04, R^{2}=-41.25 \%\right)$, or body mass index $(\beta=0.12,95 \%$ $\mathrm{CI}-0.18$ to $0.42, R^{2}=1.36 \%$ ) (panels $\mathrm{A}-\mathrm{C}$ ) on the association between chronic plaque psoriasis and risk of having NAFLD.

\section{Risk of bias}

As shown in Supplementary Fig. 6, the ranking on the NOS was higher than eight points (i.e., low risk of bias) in four studies, equal to seven points (i.e. medium risk of bias) in 2 studies, and six or fewer points in the remaining 5 studies (i.e. high risk of bias). Publication bias was estimated unlikely because no asymmetry of the funnel plots was 
found, as formally tested by the Begg's rank correlation test $(p=0.436)$, or the Egger's regression test $(p=0.183)$ (Supplementary Fig. 7).

\section{Discussion}

Our updated meta-analysis provides evidence for a significant association between chronic plaque psoriasis and risk of prevalent NAFLD, as detected by ultrasonography or ICD$9 / 10$ codes. Our meta-analysis involves a total of 15 observational cross-sectional studies with aggregate data on more than 1.7 million individuals, i.e., 249,933 psoriatic patients (49\% with NAFLD) and 1,491,402 non-psoriatic healthy controls (36\% with NAFLD). Specifically, we found that patients with chronic plaque psoriasis had a nearly twofold higher odds of prevalent NAFLD compared to non-psoriatic healthy controls. The magnitude of this risk remained essentially unchanged even when the comparison was stratified by study country, modality of NAFLD diagnosis, NOS quality scale or degree of covariate adjustment. Notably, the risk of NAFLD appeared to increase further with the severity of psoriasis, given that psoriatic patients with NAFLD had significantly higher mean PASI score than their counterparts without NAFLD. Furthermore, the risk of NAFLD also tended to be higher among patients with PsA than among those with skin psoriasis alone.

Our findings corroborate and extend the results of two previous smaller meta-analyses (published in 2015 and 2019) $[9,10]$. These two meta-analyses included a lower number of observational studies (from 6 to 9 cross-sectional or case-control studies) with a smaller overall sample size. In the first meta-analysis including 6 case-control studies, Candia et al. reported that psoriatic patients had 2.1-fold increased odds of prevalent NAFLD (as detected by ultrasonography or ICD-9/10 codes) compared to nonpsoriatic individuals ( $n=6$ studies included; pooled randomeffects OR 2.15, 95\% CI 1.57-2.94; $I^{2}=74 \%$ ) [9]. This risk appeared to be comparable between patients with mild psoriasis and those with moderate to severe psoriasis. In second meta-analysis that incorporated 9 cross-sectional or case-control studies, Phan et al. also showed an almost doubled risk of having NAFLD (as detected by ultrasonography or ICD-9/10 codes) in patients with psoriasis compared to non-psoriatic controls $(n=6$ studies included; pooled random-effects OR $1.95,95 \%$ CI $1.35-2.83 ; I^{2}=91 \%$ ) [10]. In this meta-analysis, the authors failed to show any significant association between the severity of psoriasis (assessed by PASI score) and NAFLD, although the odds of NAFLD was marginally (but not significantly) higher in patients with PsA than in those with skin psoriasis alone ( $n=3$ studies; pooled random-effects OR $3.74,95 \%$ CI $0.85-16.3 ; I^{2}=54 \%$ ).
Collectively, therefore, compared to the aforementioned two smaller meta-analyses, we have almost doubled the number of eligible studies, increased the overall sample size, and performed subgroup analyses and meta-regressions to test the robustness of the observed associations. That said, the evidence from this and the two previously published meta-analyses supports the existence of a significant association between the presence and severity of psoriasis and the risk of prevalent NAFLD.

To date, the precise underlying mechanisms linking chronic plaque psoriasis and NAFLD are complex and poorly understood. Psoriasis and NAFLD may share complex, multi-factorial and overlapping metabolic abnormalities. A cluster of interconnected features defining the metabolic syndrome are known to be closely associated with chronic plaque psoriasis, including abdominal obesity, atherogenic dyslipidaemia, hypertension, insulin resistance and dysglycaemia [34]. In particular, insulin resistance plays a pathogenic role in the development of NAFLD, as it promotes an increased free fatty acid flux from visceral adipose tissue into the liver, thereby increasing hepatic de novo lipogenesis [34]. On the other hand, hepatic fat accumulation may further aggravate systemic and hepatic insulin resistance and promote increased hepatic glucose production [35]. Additionally, overlapping mechanisms of the so-called "metaflammation" may also contribute to the complex link between psoriasis and NAFLD. Indeed, we found that both psoriasis severity and PsA correlated with an increased risk of prevalent NAFLD. The secretion of pro-inflammatory, pro-thrombotic and oxidative stress mediators in both psoriatic skin and adipose tissue might act systemically and promote insulin resistance and other metabolic derangements that promote the development and progression of NAFLD. For example, tumour necrosis factor-alfa, as well as interleukins (IL)-1, IL-2, IL-6 and IL-17 may contribute to psoriatic plaque development, as well as impaired glucose metabolism and insulin resistance in both hepatocytes and adipocytes [36]. However, the link between NAFLD and psoriasis is more complex than previously believed. Indeed, it is also possible to hypothesize that NAFLD (especially in its more advanced forms) may release multiple pro-inflammatory and pro-oxidant mediators (which is also one of the most important mechanisms by which NAFLD may contribute to the development of CVD and other extra-hepatic complications) [4-7] that may adversely influence the severity of psoriasis by increased keratinocyte proliferation, increased inflammation, and up-regulation of various vascular adhesion molecules [8].

We believe that the observed association between psoriasis and NAFLD is clinically relevant and may have practical implications. Whilst phototherapy or topical treatments are not usually associated with significant abnormalities in metabolic parameters and serum liver enzymes, specific 
Table 2 Characteristics of the eligible studies evaluating the psoriasis severity (assessed by PASI score) in psoriatic patients with and without NAFLD

\begin{tabular}{|c|c|c|c|c|c|c|c|c|}
\hline $\begin{array}{l}\text { References } \\
\text { (PMID) }\end{array}$ & Country & Patients, $n$ & Age, years ${ }^{b}$ & PASI $^{\mathrm{b}}$ & $\begin{array}{l}\text { Diagnosis of } \\
\text { NAFLD }\end{array}$ & $\begin{array}{l}\text { Prevalence } \\
\text { of NAFLD, } \\
n(\%)\end{array}$ & $\begin{array}{l}\text { Risk of NAFLD } \\
\text { in PsA vs. PsO } \\
\text { (OR } 95 \% \mathrm{CI})\end{array}$ & $\begin{array}{l}\text { Mean PASI dif- } \\
\text { ference in patients } \\
\text { with NAFLD (IV, } \\
95 \% \mathrm{CI})^{\mathrm{a}}\end{array}$ \\
\hline $\begin{array}{l}\text { Gisondi } \\
\text { et al. [21] } \\
(19560226)\end{array}$ & Italy & 130 & $51.2 \pm 13.4$ & $13.1 \pm 11.0$ & US & $61(47)$ & $1.02(0.51-2.03)$ & $7.90(4.67-11.13)$ \\
\hline $\begin{array}{l}\text { Miele et al. [31] } \\
(19664838)\end{array}$ & Italy & 84 & $50.1 \pm 15$ & $16.4 \pm 10.0$ & $\begin{array}{l}\text { US, biopsy ( } 5 \\
\text { cases) }\end{array}$ & $84(59.2)$ & $\begin{array}{l}3.53(0.98- \\
12.77)\end{array}$ & $\begin{array}{l}2.00(-1.96 \text { to } \\
5.96)\end{array}$ \\
\hline $\begin{array}{l}\text { Madanagob- } \\
\text { alane and } \\
\text { Anandan [23] } \\
(22672067)\end{array}$ & India & 333 & $46.3 \pm 11.5$ & $6.5 \pm 10.8$ & US and TE & $58(17.4)$ & $3.71(1.89-7.30)$ & $\begin{array}{l}2.20(-0.62 \text { to } \\
5.02)\end{array}$ \\
\hline $\begin{array}{l}\text { Roberts } \\
\quad \text { et al. [33] } \\
(25521607)\end{array}$ & United States & 103 & $52.7 \pm 12$ & $5.2 \pm 4.9$ & $\begin{array}{l}\text { TE, biopsy ( } 52 \\
\text { cases) }\end{array}$ & $48(46.6)$ & NR & $2.21(0.64-3.79)$ \\
\hline $\begin{array}{l}\text { Gisondi } \\
\text { et al. [22] } \\
(26537011)\end{array}$ & Italy & 124 & $55.0 \pm 12.0$ & $13.0 \pm 10.0$ & US & $55(44)$ & $1.00(0.52-1.95)$ & $8.40(5.14-11.66)$ \\
\hline $\begin{array}{l}\text { Narayanasamy } \\
\text { et al. [32] } \\
(28053681)\end{array}$ & India & 250 & $44.7 \pm 12.0$ & $27.8 \pm 13.5$ & US and TE & $113(45.2)$ & NR & $9.69(6.29-13.09)$ \\
\hline $\begin{array}{l}\text { Awosika } \\
\text { et al. [20] } \\
(29942422)\end{array}$ & United States & 101 & $44.2 \pm 13.6$ & $4.8 \pm 2.1$ & US & $21(21.2)$ & NR & $\begin{array}{l}0.72(-0.20 \text { to } \\
1.64)\end{array}$ \\
\hline $\begin{array}{l}\text { Magdaleno } \\
\text { Tapial } \\
\text { et al. [30] } \\
(31731325)\end{array}$ & Spain & 71 & $46.7 \pm 14$ & $14.4 \pm 6.5$ & US and TE & $37(52.1)$ & $2.15(0.64-7.21)$ & $1.30(0.30-2.30)$ \\
\hline
\end{tabular}

$N R$ not reported, $O R$ odds ratio, $U S$ ultrasonography, $I V$ interval variable, $T E$ transitional elastography, PASI Psoriasis Area Severity Index, $P s A$ psoriatic arthritis

${ }^{\mathrm{a}}$ Median (IQR)

${ }^{\mathrm{b}}$ Mean \pm standard deviation

pharmacological treatments may, instead, adversely affect metabolic comorbidities, including NAFLD. In this regard, it is also important to highlight that patients with moderate-to-severe psoriasis are often treated with potentially hepatotoxic drugs, such as methotrexate or cyclosporine. Methotrexate may induce histological changes in the liver that are comparable to those observed in NAFLD [37, 38]. In addition, psoriatic patients with type 2 diabetes or obesity are at higher risk of developing hepatic fibrosis during methotrexate treatment compared with their counterparts without coexisting metabolic comorbidities [8]. Cyclosporine is metabolized by the hepatic cytochrome P450 system and may worsen glycaemic control in patients with type 2 diabetes, exacerbate arterial hypertension and predispose to atherogenic dyslipidemia [39]. For this reason, cyclosporine should be used cautiously in psoriatic patients with NAFLD or other metabolic comorbidities. Conversely, the newer biologic drugs do not seem to affect negatively liver steatosis and metabolic parameters, although a weight gain has been reported with the long-term use of some TNF- $\alpha$ antagonists [40]. IL-17 inhibitors are being investigated as a potential therapeutic targeting therapy for patients with NAFLD and psoriasis [41]. Consequently, we believe that the presence of NAFLD in psoriatic patients should be always considered when choosing pharmacological treatment, as some drugs for psoriasis may be potentially hepatotoxic.

Our findings also imply that psoriatic patients might be screened with liver ultrasonography especially when there are also coexisting metabolic features associated with NAFLD (e.g., obesity, atherogenic dyslipidemia or dysglycemia). However, the optimal method of screening in these patients is currently unknown. In view of the intrinsic limitations of measurement of serum liver enzymes alone as initial screening test for NAFLD, we believe that liver ultrasonography combined with the use of non-invasive biomarkers of advanced fibrosis (such as NAFLD fibrosis score [NFS] or Fibrosis-4 score [FIB-4]) or, alternatively, vibration-controlled transient elastography (Fibroscan) 
Risk of prevalent NAFLD in psoriatic patients vs. healthy controls

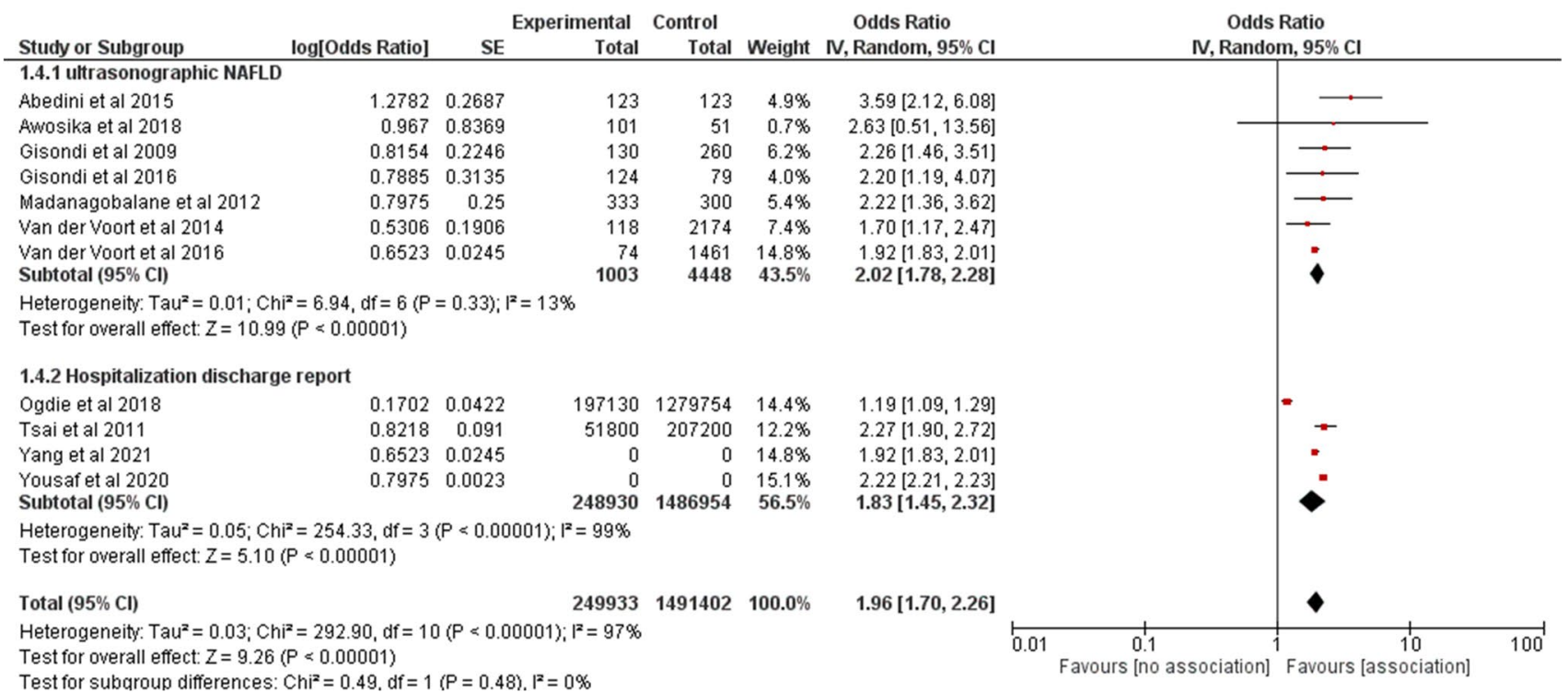

Fig. 2 Forest plot and pooled estimates of the effect of chronic plaque psoriasis on the risk of prevalent NAFLD in 11 eligible studies, stratified by NAFLD diagnosis (ultrasonography vs. International Classification of Diseases codes)

\section{Mean PASI score in psoriatic patients with NAFLD vs. without NAFLD}

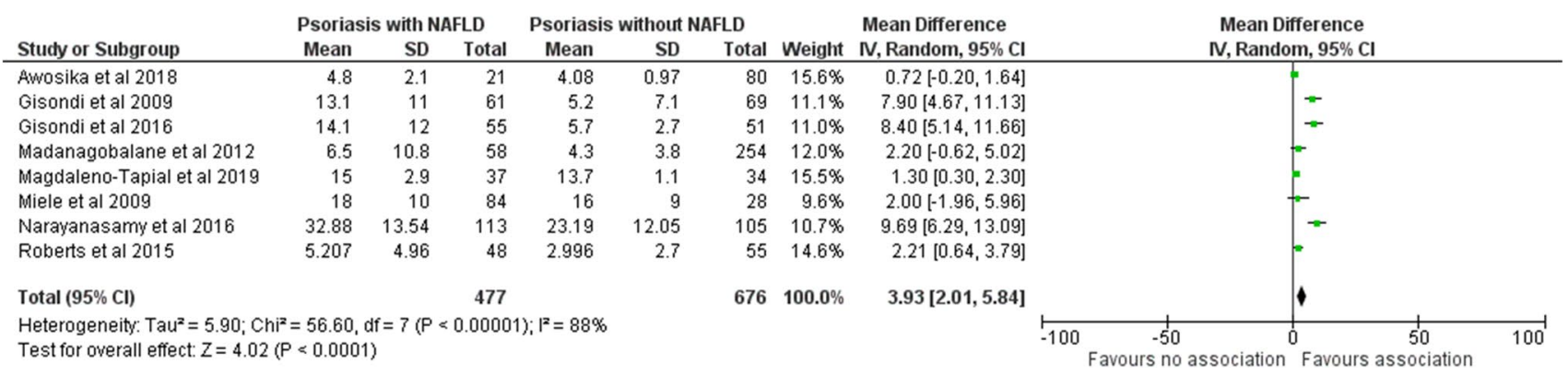

Fig. 3 Forest plot and pooled estimates of PASI score in psoriatic patients with and without NAFLD ( $n=8$ studies included). Data are expressed as weighted mean difference (WMD) and $95 \%$ confidence intervals

\section{Effect of psoriatic arthritis on the risk of prevalent NAFLD}

\begin{tabular}{|c|c|c|c|c|c|c|c|c|c|c|}
\hline Study or Subgroup & \multicolumn{2}{|c|}{ PSA } & \multicolumn{2}{|c|}{ PSO } & Weight & $\begin{array}{c}\text { Odds Ratio } \\
\text { M-H, Random, } 95 \% \mathrm{Cl}\end{array}$ & \multicolumn{3}{|c|}{$\begin{array}{c}\text { Odds Ratio } \\
\text { M-H, Random, } 95 \% \mathrm{Cl}\end{array}$} & \\
\hline Gisondi et al 2009 & 33 & 70 & 28 & 60 & $23.5 \%$ & $1.02[0.51,2.03]$ & & & & \\
\hline Gisondi et al 2016 & 27 & 52 & 55 & 106 & $24.1 \%$ & $1.00[0.52,1.95]$ & & & & \\
\hline Madanagobalane et al 2012 & 21 & 47 & 37 & 207 & $23.8 \%$ & $3.71[1.89,7.30]$ & & & & \\
\hline Magdaleno-Tapial et al 2019 & 9 & 14 & 26 & 57 & $14.8 \%$ & $2.15[0.64,7.21]$ & & & & \\
\hline Miele et al 2009 & 25 & 28 & 59 & 84 & $13.8 \%$ & $3.53[0.98,12.77]$ & & & & \\
\hline Total $(95 \% \mathrm{CI})$ & & 211 & & 514 & $100.0 \%$ & $1.83[0.98,3.43]$ & & & & \\
\hline Total events & 115 & & 205 & & & & & & & \\
\hline $\begin{array}{l}\text { Heterogeneity: } \operatorname{Tau}^{2}=0.31 ; \mathrm{Cl} \\
\text { Test for overall effect: } Z=1.89\end{array}$ & $\begin{array}{l}P=11.05 \\
P=0.06)\end{array}$ & $\mathrm{df}=4$ & $(P=0.03$ & $; I^{2}=6$ & $4 \%$ & & 0.01 & $\begin{array}{c}0.1 \\
\text { Favours [PSO] }\end{array}$ & Favours [PSA] & 100 \\
\hline
\end{tabular}

Fig. 4 Forest plot and pooled estimates of the effect of psoriatic arthritis (PsA) on the risk of prevalent NAFLD ( $n=6$ studies included) 
might be fruitful as first-line choice in identifying psoriatic patients with NAFLD and advanced fibrosis, in order to refer to a hepatologist [8]. In addition, we also believe that all psoriatic patients with NAFLD should be also followed routinely to assess the development of liver-related, metabolic and CVD complications [8].

This meta-analysis has some important limitations that should be mentioned. First, the cross-sectional design of the studies precludes us from establishing causality and temporality for the observed associations. Second, the overall quality of the included studies is not consistently high and some of the eligible studies reported incomplete adjustments for potential confounding factors, thus the possibility of residual confounding by unmeasured factors cannot be ruled out. The degree of adjustment performed in most of the eligible studies is limited, as some studies did not adjust for BMI and, most importantly, no studies adjusted also for insulin resistance or plasma inflammatory biomarkers. Thirdly, even though we used the DerSimonian-Laird random-effects model, the interpretation of some results of this meta-analysis should require some caution in view of the high heterogeneity observed. However, it is important to underline that we found a very low heterogeneity $\left(I^{2}=13 \%\right)$ when we pooled the studies examining the association between psoriasis and risk of NAFLD, where the diagnosis of NAFLD was made with ultrasonography. Another potential limitation of our metaanalysis is that the majority of the eligible studies used imaging techniques (mostly ultrasonography) or ICD-9/10 codes that are not always reported by physicians during their daily clinical practice. Indeed, only two studies used liver biopsy, which is the reference method for diagnosing and staging NAFLD, but exclusively in a number of patients that was too small for making any statistical analysis. Thus, it is currently uncertain if psoriatic patients are also more likely to have more advanced forms of NAFLD (i.e., NASH with varying levels of fibrosis) compared with non-psoriatic controls.

Although these limitations, our meta-analysis has some important strengths. This review summarizes data from large studies that are likely to reflect people with chronic plaque psoriasis usually seen in clinical practice. We could not definitely exclude a selective reporting bias, but we believe that our search has made such bias unlikely. Finally, visual inspection of the funnel plot did not show any publication bias.

In conclusion, this updated meta-analysis of more than 1.7 million individuals of different countries shows that psoriasis is significantly associated with a nearly twofold increased odds of prevalent NAFLD. This risk parallels the underlying severity of chronic plaque psoriasis. Given the observational design of the eligible studies and the fact that most eligible studies used ultrasonography for detecting
NAFLD, the findings of this meta-analysis pave the way for novel large, prospective, histologically based studies. Mechanistic studies are also needed to better elucidate the existing but complex link between psoriasis and NAFLD.

Supplementary Information The online version contains supplementary material available at https://doi.org/10.1007/s40618-022-01755-0.

Funding Fondazione Cariplo, Fondazione Veronesi, Impact of COVID19 infection on patients affected by inflammatory skin diseases on immunosuppressive therapies (COVISKIN); ID 1833073 rif. 20201363. GT is supported in part by grants from the University School of Medicine of Verona, Verona, Italy.

Availability of data and material Not applicable.

Code availability Not applicable.

\section{Declarations}

Conflict of interest F. Bellinato: none declared; P. Gisondi: none declared; A. Mantovani: none declared; G. Girolomoni: none declared; G. Targher: none declared.

Ethics approval Not applicable.

Informed consent Not applicable.

Consent to participate Not applicable.

Consent for publication Not applicable.

Open Access This article is licensed under a Creative Commons Attribution 4.0 International License, which permits use, sharing, adaptation, distribution and reproduction in any medium or format, as long as you give appropriate credit to the original author(s) and the source, provide a link to the Creative Commons licence, and indicate if changes were made. The images or other third party material in this article are included in the article's Creative Commons licence, unless indicated otherwise in a credit line to the material. If material is not included in the article's Creative Commons licence and your intended use is not permitted by statutory regulation or exceeds the permitted use, you will need to obtain permission directly from the copyright holder. To view a copy of this licence, visit http://creativecommons.org/licenses/by/4.0/.

\section{References}

1. Parisi R, Iskandar IYK, Kontopantelis E et al (2020) National, regional, and worldwide epidemiology of psoriasis: systematic analysis and modelling study. BMJ 369:m1590

2. Gisondi P, Bellinato F, Girolomoni G, Albanesi C (2020) Pathogenesis of chronic plaque psoriasis and its intersection with cardio-metabolic comorbidities. Front Pharmacol 11:117

3. Targher G (2020) What's past is prologue: history of nonalcoholic fatty liver disease. Metabolites 10:397

4. Mantovani A, Scorletti E, Mosca A, Alisi A, Byrne CD, Targher G (2020) Complications, morbidity and mortality of nonalcoholic fatty liver disease. Metabolism 111S:154170 
5. Anstee QM, Mantovani A, Tilg H, Targher G (2018) Risk of cardiomyopathy and cardiac arrhythmias in patients with nonalcoholic fatty liver disease. Nat Rev Gastroenterol Hepatol 15:425-439

6. Mantovani A, Petracca G, Beatrice G, Csermely A, Lonardo A, Schattenberg JM, Tilg H, Byrne CD, Targher G (2020) Nonalcoholic fatty liver disease and risk of incident chronic kidney disease: an updated meta-analysis. Gut. 71(1):156-162

7. Mantovani A, Petracca G, Beatrice G, Csermely A, Tilg H, Byrne CD, Targher G (2021) Non-alcoholic fatty liver disease and increased risk of incident extrahepatic cancers: a meta-analysis of observational cohort studies. Gut. gutjnl-2021-324191

8. Mantovani A, Gisondi P, Lonardo A, Targher G (2016) Relationship between non-alcoholic fatty liver disease and psoriasis: a novel hepato-dermal axis? Int J Mol Sci 17:217

9. Candia R, Ruiz A, Torres-Robles R, Chávez-Tapia N, MéndezSánchez N, Arrese M (2015) Risk of non-alcoholic fatty liver disease in patients with psoriasis: a systematic review and metaanalysis. J Eur Acad Dermatol Venereol 29:656-662

10. Phan K, Onggo J, Charlton O, Smith SD (2019) Relationship between psoriasis and non-alcoholic fatty liver disease-updated systematic review and adjusted meta-analysis. Australas J Dermatol 60:e352-e355

11. Moher D, Liberati A, Tetzlaff J et al (2009) Preferred reporting items for systematic reviews and meta-analyses: the PRISMA statement. PLoS Med 6:e1000097

12. Stroup DF, Berlin JA, Morton SC et al (2000) Meta-analysis of observational studies in epidemiology: a proposal for reporting. Meta-Analysis of Observational Studies in Epidemiology (MOOSE) group. JAMA 283:2008-2012

13. Taylor W, Gladman D, Helliwell P et al (2006) Classification criteria for psoriatic arthritis; development of new criteria from a large international study. Arthritis Rheum 54:2665-2673

14. Chalasani N, Younossi Z, Lavine JE, Diehl AM, Brunt EM, Cusi $\mathrm{K}$ et al, American Gastroenterological Association, American Association for the Study of Liver Diseases, American College of Gastroenterology (2012) The diagnosis and management of nonalcoholic fatty liver disease: practice guideline by the American Gastroenterological Association, American Association for the Study of Liver Diseases, and American College of Gastroenterology. Gastroenterology 142:1592-1609

15. Wells GA, Shea B, O'Connell D et al (2011) The NewcastleOttawa Scale (NOS) for assessing the quality of case-control studies in meta-analyses. Eur J Epidemiol 25:603-605. http://www. ohri.ca/programs/clinical_epidemiology/oxford.asp. Accessed 1 Dec 2021

16. Higgins JP, Thompson SG (2002) Quantifying heterogeneity in a meta-analysis. Stat Med 21:1539-1558

17. Begg CB, Mazumdar M (1994) Operating characteristics of a rank correlation test for publication bias. Biometrics 50:1088-1101

18. Egger M, Smith GD, Phillips AN (1997) Meta-analysis: principles and procedures. BMJ 315:1533-1537

19. Abedini R, Salehi M, Lajevardi V, Beygi S (2015) Patients with psoriasis are at a higher risk of developing nonalcoholic fatty liver disease. Clin Exp Dermatol 40:722-727

20. Awosika O, Eleryan MG, Rengifo-Pardo M, Doherty L, Martin LW, Ehrlich A (2018) A case-control study to evaluate the prevalence of nonalcoholic fatty liver disease among patients with moderate-to-severe psoriasis. J Clin Aesthet Dermatol 11:33-37

21. Gisondi P, Targher G, Zoppini G, Girolomoni G (2009) Non-alcoholic fatty liver disease in patients with chronic plaque psoriasis. J Hepatol 51:758-764

22. Gisondi P, Barba E, Girolomoni G (2016) Non-alcoholic fatty liver disease fibrosis score in patients with psoriasis. J Eur Acad Dermatol Venereol 30:282-287
23. Madanagobalane S, Anandan S (2012) The increased prevalence of non-alcoholic fatty liver disease in psoriatic patients: a study from South India. Australas J Dermatol 53:190-197

24. Ogdie A, Grewal SK, Noe MH, Shin DB, Takeshita J, Chiesa Fuxench ZC et al (2018) Risk of incident liver disease in patients with psoriasis, psoriatic arthritis, and rheumatoid arthritis: a population-based study. J Investig Dermatol 138:760-767

25. Tsai TF, Wang TS, Hung ST, Tsai PI, Schenkel B, Zhang M et al (2011) Epidemiology and comorbidities of psoriasis patients in a national database in Taiwan. J Dermatol Sci 63:40-46

26. van der Voort EA, Koehler EM, Dowlatshahi EA, Hofman A, Stricker BH, Janssen HL et al (2014) Psoriasis is independently associated with nonalcoholic fatty liver disease in patients 55 years old or older: results from a population-based study. J Am Acad Dermatol 70:517-524

27. van der Voort EA, Koehler EM, Nijsten T, Stricker BH, Hofman A, Janssen HL et al (2016) Increased prevalence of advanced liver fibrosis in patients with psoriasis: a cross-sectional analysis from the Rotterdam study. Acta Derm Venereol 96:213-217

28. Yang JJ, Nguyen KA, Fleischman MW, Aly O, Cheng K (2021) Psoriasis in liver disease: associations beyond nonalcoholic fatty liver disease. J Am Acad Dermatol S0190-9622:00562-00564

29. Yousaf A, Raiker R, Davis SM, Gayam S, Zinn Z (2020) Association between psoriasis, psoriatic arthritis and gastrointestinal disease: an exploratory nationwide inpatient sample analysis. Wien Klin Wochenschr. https://doi.org/10.1007/s00508-020-01740-8

30. Magdaleno-Tapial J, Valenzuela-Oñate C, Ortiz-Salvador JM, Martínez-Doménech Á, García-Legaz-Martínez M, AlonsoCarpio M et al (2020) Prevalence of non-alcoholic fatty liver and liver fibrosis in patients with moderate-severe psoriasis: a crosssectional cohort study. Australas J Dermatol 61:105-109

31. Miele L, Vallone S, Cefalo C, La Torre G, Di Stasi C, Vecchio FM et al (2009) Prevalence, characteristics and severity of nonalcoholic fatty liver disease in patients with chronic plaque psoriasis. J Hepatol 51:778-786

32. Narayanasamy K, Sanmarkan AD, Rajendran K, Annasamy C, Ramalingam S (2016) Relationship between psoriasis and nonalcoholic fatty liver disease. Prz Gastroenterol 11:263-269

33. Roberts KK, Cochet AE, Lamb PB, Brown PJ, Battafarano DF, Brunt EM, Harrison SA (2015) The prevalence of NAFLD and NASH among patients with psoriasis in a tertiary care dermatology and rheumatology clinic. Aliment Pharmacol Ther 41:293-300

34. Wenk KS, Arrington KC, Ehrlich A (2011) Psoriasis and nonalcoholic fatty liver disease. J Eur Acad Dermatol Venereol 25:383-391

35. Eslam M, Newsome PN, Sarin SK, Anstee QM, Targher G, Romero-Gomez M et al (2020) A new definition for metabolic dysfunction-associated fatty liver disease: an international expert consensus statement. J Hepatol 73:202-209

36. Lim S, Kim JW, Targher G (2021) Links between metabolic syndrome and metabolic dysfunction-associated fatty liver disease. Trends Endocrinol Metab 32:500-514

37. Davidovici BB, Sattar N, Prinz J, Puig L, Emery P, Barker JN et al (2010) Psoriasis and systemic inflammatory diseases: potential mechanistic links between skin disease and co-morbid conditions. J Investig Dermatol 130:1785-1796

38. Gisondi P, Bellinato F, Bruni M, De Angelis G, Girolomoni G (2020) Methotrexate vs secukinumab safety in psoriasis patients with metabolic syndrome. Dermatol Ther 33:e14281

39. Chiricozzi A, Gisondi P, Girolomoni G (2019) The pharmacological management of patients with comorbid psoriasis and obesity. Expert Opin Pharmacother 20:863-872

40. Gerdes S, Pinter A, Papavassilis C, Reinhardt M (2020) Effects of secukinumab on metabolic and liver parameters in plaque psoriasis patients. J Eur Acad Dermatol Venereol 34:533-541 
41. Thaçi D, Schäkel K, Cornberg M, Sieder C, Bachhuber T, Hillmann E et al. Secukinumab in psoriasis patients with nonalcoholic fatty liver disease: study design and objectives of the PINPOINT trial. In: Poster session presented at: 29th European Academy of Dermatology and Venereology, 29-31st October 2020, Virtual Congress
Publisher's Note Springer Nature remains neutral with regard to jurisdictional claims in published maps and institutional affiliations. 